\title{
Review of: "Single-Round Remodeling of the Active Site of an Artificial Metalloenzyme using an Ultrahigh- Throughput Double Emulsion Screening Assay"
}

Ying-Wu Lin

Potential competing interests: The author(s) declared that no potential competing interests exist.

In this report, Dittrich, Ward and co-workers developed an ultrahigh-throughput assay for the directed evolution of an artificial deallylase using double-emulsion droplet (DE)-microfluidics. Moreover, they verified the DE-based assay by screen a library containing 400 variants. The concept was well presented and the results were well discussed. Therefore, this technique is acceptable for quick publication in bioRxiv. Several minor comments are listed below:

1. It is difficult to understand "Single-Round Remodeling of the Active Site" in the title, as it was not mentioned or discussed in the main text. To this referee, double-emulsion droplets (DEs) construct a micro-environment for the ArM, instead of the metal active site. Therefore, the title is suggested to be "Directed Evolution of an Artificial Metalloenzyme using ..."

2. Similarly, in the Conclusion section (line 200), "remodeling of an active site" might be revised accordingly.

3. In the Introduction section (line 39), "make it" should be "making it" or "and make it".

4. In Figure S4, it is also helpful to present an overall structure of Sav to show the metal active site and the position of $\mathrm{S} 112$ and $\mathrm{K} 121$. 